\title{
TOPOLOGICAL SOLUTIONS OF $\eta$-GENERALIZED VECTOR VARIATIONAL-LIKE INEQUALITY PROBLEMS
}

\author{
SATISH KUMAR, ANKIT GUPTA, PANKAJ KUMAR GARG AND RATNA DEV SARMA
}

\begin{abstract}
In this paper, we discuss several variants of the $\eta$-generalized vector variational-like inequality problem and provide existence theorems for their solutions via a topological approach. Several topological concepts like compactness, closedness, net theory and admissibility of function space topology are used for obtaining the main results. Finally, we give some topological properties of the solution set so obtained.
\end{abstract}

\section{INTRODUCTION}

In 1980, F. Gianessi [4] extended the concept of classical variational inequality (introduced by Stampacchia [23]) to vector variational inequality (VVI, in short) for vector valued functions in the setting of finite-dimensional Euclidean spaces. Further, VVI has been extended in various directions, in particular, the vector variational-like inequalities (VVLI, in short) $[3,8,9,18,20]$. VVI and their generalizations have been used extensively to solve vector optimization problems. Several researchers have established various relations between vector variational inequalities and vector optimization problems [12,14,24,28].

In one direction, the concept of variational inequality was extended by Hanson [7] by introducing invex function (a generalization of convex function). Weir and Mond [25] and Noor [19] have studied some basic properties of preinvex and $\alpha$-preinvex functions, respectively, along with their role in variational-like inequality problems and optimization problems. By assuming the condition of pseudoinvexity, Ruiz-Garzon et al. [20] have established some relations between vector variational-like inequality problems and optimization problems. In [8-10], Khan and others studied several variants of vector variational-like inequalities in the framework of Banach spaces. In 2017, Salahuddin [21] provided existence results for the solution of general set-valued vector variational inequalities. In the same year, Li and $\mathrm{Yu}$ [16] introduced a class of generalized invex functions, namely $(\alpha-\rho-\eta)$-invex functions and provided the existence results for two types of vector variational-like inequalities. On the other hand, in 2018, Salahuddin [22] obtained the existence results for the solution of vector variational inequality problems by using sequentially continuous mapping. Recently, Gupta et al. [5] provided existence theorems for the solution of generalized non-linear vector variational-like

$M S C$ (2020): primary 49J40, 54H99 ; secondary 58E35.

Keywords: $\eta$-Generalized vector variational-like inequality, KKM mapping; set-valued function, topological vector space, compactness. 
inequality problems by using topological approach.

Variational-like inequalities have a wide range of applications, making it an interesting discipline for research. The flow equilibrium problem on a network using vector variational inequality has been discussed in [29]. Further, application of variational-like inequality in fuzzy setting for the optimization problem is discussed in [26]. Similar studies are available in the literature [17,27,30]. Motivated by these studies, here we study a generalized form of a vector variational-like inequality problem.

In [13], Lee et al. discussed the solvability of generalized weak vector variationallike inequalities (GWVVLI) in Banach spaces (reflexive Banach spaces) by using the Browder fixed point theorems and monotonicity of mappings and, in [15], Li et al. introduced a class of $\eta$-generalized vector variational-like inequalities $(\eta$ GVVLI) for Hausdorff topological vector spaces and gave two existence results for solution to the $\eta$-GVVLI problem under the assumption of $\eta$-hemicontinuity; in one result the compactness of $K$ is considered, while in the other it is not so.

In the present paper, we consider a couple of $\eta$-GVVLI problems and prove the existence results for these problems in topological vector spaces using topological approach. We use the concept of closedness, compactness, and net theory along with the admissibility of a function space to obtain our results. The authors have found so far that the concept of admissibility of function spaces is not used extensively in the existing literature to obtain such results.

In the following, we will define two variants of the $\eta$-GVVLI problem:

Let $X$ and $Y$ be two topological vector spaces and $\mathcal{C}(X, Y)$ be the space of all continuous linear mappings from the space $X$ to the space $Y$. Let $K \subseteq X$ be nonempty, closed and convex. Further, let $T: K \times K \times[0,1] \rightarrow \mathcal{C}(X, Y)$ be a single-valued map defined by $T(x, z, \lambda)=T_{\lambda x+(1-\lambda) z}$ and $\eta: K \times K \rightarrow X$, $f: K \times K \rightarrow Y$ be two bifunctions.

- $\eta$-generalized vector variational-like inequality problem I ( $\eta$-GVVLIP (I)): If $C$ is a closed convex pointed cone in $Y$ with $\operatorname{int} C \neq \emptyset$, then $\eta$-GVVLIP (I) is to find $x_{0} \in K$ such that there exist $z \in K, \lambda \in[0,1]$ satisfying

$$
T\left(x_{0}, z, \lambda\right)\left(\eta\left(y, x_{0}\right)\right)+f\left(y, x_{0}\right) \notin-\operatorname{int} C \quad \forall y \in K .
$$

- $\eta$-generalized vector variational-like inequality problem II ( $\eta$-GVVLIP (II)): If $C: K \rightrightarrows Y$ is a set-valued map such that for each $x \in K, C(x)$ is a closed convex pointed cone in $Y$ with int $C(x) \neq \emptyset$, then $\eta$-GVVLIP (II) is to find $x_{0} \in K$ such that there exist $z \in K, \lambda \in[0,1]$ satisfying

$$
T\left(x_{0}, z, \lambda\right)\left(\eta\left(y, x_{0}\right)\right)+f\left(y, x_{0}\right) \notin-\operatorname{int} C\left(x_{0}\right) \quad \forall y \in K .
$$

By considering a mapping $T: K \times K \times(0,1] \rightarrow \mathcal{C}(X, Y)$ instead of $T: K \times$ $K \times[0,1] \rightarrow \mathcal{C}(X, Y)$ as in the above problems, we define two other variants of the $\eta$-GVVLI problem:

- $\eta$-generalized vector variational-like inequality problem III ( $\eta$-GVVLIP (III)): If $C$ is a closed convex pointed cone in $Y$ with $\operatorname{int} C \neq \emptyset$, then for some fixed $\lambda \in(0,1], \eta$-GVVLIP (III) is to find $x_{0} \in K$ such that there exist $z \in K$ satisfying

$$
T\left(x_{0}, z, \lambda\right)\left(\eta\left(y, x_{0}\right)\right)+f\left(y, x_{0}\right) \notin-\operatorname{int} C \quad \forall y \in K
$$


- $\eta$-generalized vector variational-like inequality problem IV ( $\eta$-GVVLIP (IV): If $C: K \rightrightarrows Y$ is a set-valued map such that for each $x \in K, C(x)$ is a closed convex pointed cone in $Y$ with $\operatorname{int} C(x) \neq \emptyset$, then for some fixed $\lambda \in(0,1]$, $\eta$-GVVLIP (IV) is to find $x_{0} \in K$ such that there exist $z \in K$ satisfying

$$
T\left(x_{0}, z, \lambda\right)\left(\eta\left(y, x_{0}\right)\right)+f\left(y, x_{0}\right) \notin-\operatorname{int} C\left(x_{0}\right) \quad \forall y \in K .
$$

The rest of the paper is organized in the following way: In Section 2, we recall some preliminaries required in the paper. In Section 3, we prove existence theorems for solutions to the $\eta$-GVVLIP (I) and $\eta$-GVVLIP (II). We then give an example to illustrate our results. Finally, we provide some properties of the solution sets so obtained.

\section{Preliminaries}

In this section, we recall some definitions and basic results which will be used later to obtain the main results.

Definition 2.1. Suppose $F: X \rightrightarrows Y$ is a set-valued map from $X$ to $Y$. The graph of $F$, denoted by $\mathcal{G}(F)$, is

$$
\mathcal{G}(F)=\{(x, y) \in X \times Y \mid x \in X, y \in F(x)\} .
$$

Definition 2.2. ([2]) Let $U$ be a nonempty subset of a topological vector space $X$. A set-valued map $F: U \rightrightarrows X$ is called a KKM-mapping if for every nonempty finite set $\left\{u_{1}, u_{2}, \ldots, u_{n}\right\}$ of $U$, we have

$$
\operatorname{co}\left\{u_{1}, u_{2}, \ldots, u_{n}\right\} \subseteq \bigcup_{i=1}^{n} F\left(u_{i}\right),
$$

where $\operatorname{co}\left\{u_{1}, u_{2}, \ldots, u_{n}\right\}$ denotes the convex hull of $u_{1}, u_{2}, \ldots, u_{n}$.

The following result is taken from [2].

Lemma 2.3. (KKM-Theorem) If $U$ is a nonempty subset of a topological vector space $X$ and $F: U \rightrightarrows X$ is a KKM-mapping such that for every $u \in U$, $F(u)$ is a closed subset of $X$ and for at least one $u \in U, F(u)$ is compact, then $\bigcap_{u \in U} F(u) \neq \emptyset$.

Definition 2.4. $([1,6])$ Let $\left(Y, \mu_{1}\right)$ and $\left(Z, \mu_{2}\right)$ be two topological spaces. Let $\mathcal{C}(Y, Z)$ be the space of all continuous mappings from $Y$ to $Z$. A topology $\tau$ on $\mathcal{C}(Y, Z)$ is called admissible, if the evaluation map e $: \mathcal{C}(Y, Z) \times Y \rightarrow Z$, defined by $e(f, y)=f(y)$, is continuous.

Lemma 2.5. ([6]) A function space topology on $\mathcal{C}(X, Y)$, the collection of continuous mappings from the space $X$ to the space $Y$, is admissible if and only if, for any net $\left\{f_{n}\right\}_{n \in D_{1}}$ in $\mathcal{C}(X, Y)$, the convergence of $\left\{f_{n}\right\}_{n \in D_{1}}$ to $f$ implies the continuous convergence of $\left\{f_{n}\right\}_{n \in D_{1}}$ to $f$. That is, if $\left\{f_{n}\right\}_{n \in D_{1}}$ converges to $f$ in $\mathcal{C}(X, Y)$ and $\left\{x_{m}\right\}_{m \in D_{2}}$ is any net in $X$ converging to $x \in X$, then $\left\{f_{n}\left(x_{m}\right)\right\}_{(n, m) \in D_{1} \times D_{2}}$ converges to $f(x)$ in $Y$.

The above characterization of admissibility remains valid for the family of continuous linear mappings from $X$ to $Y$, where $X$ and $Y$ are topological vector spaces. 
Throughout the paper, $0_{X}$ and $0_{Y}$ denote the zero vectors in the space $X$ and in the space $Y$, respectively.

\section{Existence THEOREMS FOR $\eta$-GVVLIP (I) AND $\eta$-GVVLIP (II)}

Theorem 3.1. Let $\left(X, \tau_{1}\right)$ and $\left(Y, \tau_{2}\right)$ be any two topological vector spaces. Let $\mathcal{C}(X, Y)$ denote the space of all continuous linear mappings from $X$ to $Y$, equipped with an admissible topology. Let $K \subseteq X$ be a nonempty closed convex compact subset of $X$. Let $C \subseteq Y$ be a closed convex pointed cone with int $C \neq \emptyset$. Further, let $T: K \times K \times[0,1] \rightarrow \mathcal{C}(X, Y)$ be a single-valued continuous mapping. Suppose the maps $\eta: K \times K \rightarrow X$ and $f: K \times K \rightarrow Y$ are affine maps such that both are continuous in the second argument with $\eta(x, x)=0_{X}, f(x, x)=0_{Y}$ for all $x \in K$. Then, the $\eta$-GVVLIP (I) has a solution. That is, there exists $x_{0} \in K$ such that, for some $z_{0} \in K$ and for some $\lambda_{0} \in[0,1]$, the following holds

$$
T\left(x_{0}, z_{0}, \lambda_{0}\right)\left(\eta\left(y, x_{0}\right)+f\left(y, x_{0}\right)\right) \notin-\operatorname{int} C \quad \forall y \in K .
$$

Proof. Consider a set-valued map $F: K \rightrightarrows K$ defined as

$$
F(y)=\{x \in K: \exists z \in K, \exists \lambda \in[0,1] \text { s.t. } T(x, z, \lambda)(\eta(y, x))+f(y, x) \notin-\operatorname{int} C\} .
$$

Clearly, for each $y \in K, F(y)$ is nonempty as at least $y \in K$. For convenience, we divide the proof into two steps:

(i) $F$ is a KKM-map on $K$ : Let $U=\left\{u_{1}, u_{2}, \ldots, u_{m}\right\}$ be any finite subset of $K$. Let $v \in \operatorname{co}\left\{u_{1}, u_{2}, \ldots, u_{m}\right\}$ but $v \notin \bigcup_{i=1}^{m} F\left(u_{i}\right)$. Therefore, there exist $\lambda_{1} \geq 0, \lambda_{2} \geq 0, \ldots, \lambda_{m} \geq 0$ with $\sum_{i=1}^{m} \lambda_{i}=1$ and $v=\sum_{i=1}^{m} \lambda_{i} u_{i}$. Since $v \notin F\left(u_{i}\right)$, for each $i=1,2, \ldots, m$, therefore $\forall z \in K, \forall \lambda \in[0,1]$, we have $T(v, z, \lambda)\left(\eta\left(u_{i}, v\right)\right)+f\left(u_{i}, v\right) \in-\operatorname{int} C$, for each $i=1,2, \ldots, m$. Since $-\operatorname{int} C$ is a convex set and $\lambda_{i} \geq 0$ with $\sum_{i=1}^{m} \lambda_{i}=1$, therefore

$$
\sum_{i=i}^{m} \lambda_{i}\left[T(v, z, \lambda)\left(\eta\left(u_{i}, v\right)\right)+f\left(u_{i}, v\right)\right] \in-\operatorname{int} C, \quad \forall z \in K, \forall \lambda \in[0,1] .
$$

Since $\eta$ and $f$ are affine, therefore

$$
T(v, z, \lambda)\left(\eta\left(\sum_{i=1}^{m} \lambda_{i} u_{i}, v\right)\right)+f\left(\sum_{i=1}^{m} \lambda_{i} u_{i}, v\right) \in-\operatorname{int} C, \forall z \in K, \forall \lambda \in[0,1],
$$

which implies

$$
T(v, z, \lambda)(\eta(v, v))+f(v, v) \in-\operatorname{int} C, \forall z \in K, \forall \lambda \in[0,1] .
$$

But we have $\eta(v, v)=0_{X}$ and $f(v, v)=0_{Y}$, therefore $0_{Y}$ belongs to $-\operatorname{int} C$, then $0_{Y}$ belongs to int $C$, which is a contradiction as $C$ is a pointed cone in $Y$.

(ii) $F(y)$ is closed for each $y \in K$ : Let $\left\{x_{\alpha}\right\}_{\alpha \in D}$ be a net in $F(y)$ with $\left\{x_{\alpha}\right\}$ converging to $\bar{x}$ in $X$. As $K$ is closed, $\bar{x} \in K$. We have to show that $\bar{x} \in F(y)$, that is, there exist $\bar{z} \in K, \bar{\lambda} \in[0,1]$ such that $T(\bar{x}, \bar{z}, \bar{\lambda})(\eta(y, \bar{x})+f(y, \bar{x})) \notin$ -int $C$. Since $x_{\alpha} \in F(y)$, therefore, there exist nets $\left\{z_{\alpha}\right\}_{\alpha \in D},\left\{\lambda_{\alpha}\right\}_{\alpha \in D}$ such that $T\left(x_{\alpha}, z_{\alpha}, \lambda_{\alpha}\right)\left(\eta\left(y, x_{\alpha}\right)\right)+f\left(y, x_{\alpha}\right) \notin-\operatorname{int} C$. Since $z_{\alpha} \in K$ and $\mathrm{K}$ is compact, therefore there exists a subnet $\left\{z_{\alpha_{l}}\right\}_{\alpha_{l} \in D_{1}}$ of $\left\{z_{\alpha}\right\}$ such that $\left\{z_{\alpha_{l}}\right\}$ converges to some $z$ in $K$. As $\lambda_{\alpha} \in[0,1]$, by Bolzano-Weierstrass Theorem, 
there exists a subnet $\left\{\lambda_{\alpha_{m}}\right\}_{\alpha_{m} \in D_{2}}$ such that $\left\{\lambda_{\alpha_{m}}\right\}$ converges to some $\lambda \in$ $[0,1]$. Without loss of generality, suppose $z=\bar{z}$ and $\lambda=\bar{\lambda}$.

Now, we form a directed set $D_{3} \subseteq D$ in the following manner: By the virtue of the order property of the directed set $D$, for each pair $\alpha_{l}, \alpha_{m} \in D$, there exists some $\alpha_{\delta} \in D$ such that $\alpha_{\delta} \geq \alpha_{l}, \alpha_{\delta} \geq \alpha_{m}$. Let $D_{3}$ be the compilation of such $\alpha_{\delta}$ 's. It is easy to verify that $D_{3}$ is a directed set under the induced ordering of $D$.

Thus, we have subnets $\left\{x_{\alpha_{\delta}}\right\}_{\alpha_{\delta} \in D_{3}},\left\{z_{\alpha_{\delta}}\right\}_{\alpha_{\delta} \in D_{3}}$ and $\left\{\lambda_{\alpha_{\delta}}\right\}_{\alpha_{\delta} \in D_{3}}$ of $\left\{x_{\alpha}\right\}$, $\left\{z_{\alpha}\right\}$ and $\left\{\lambda_{\alpha}\right\}$, respectively, such that $\left\{x_{\alpha_{\delta}}\right\},\left\{z_{\alpha_{\delta}}\right\},\left\{\lambda_{\alpha_{\delta}}\right\}$ converge to $\bar{x} \in K$, $\bar{z} \in K$ and $\bar{\lambda} \in[0,1]$, respectively. As $T$ is continuous, $T\left(x_{\alpha_{\delta}}, z_{\alpha_{\delta}}, \lambda_{\alpha_{\delta}}\right) \rightarrow$ $T(\bar{x}, \bar{z}, \bar{\lambda})$. Also, as $\eta(y, \cdot)$ and $f(y, \cdot)$ are continuous in second argument, $\eta\left(y, x_{\alpha_{\delta}}\right) \rightarrow \eta(y, \bar{x})$ and $f\left(y, x_{\alpha_{\delta}}\right) \rightarrow f(y, \bar{x})$. Since the function space has admissible topology, therefore

$$
T\left(x_{\alpha_{\delta}}, z_{\alpha_{\delta}}, \lambda_{\alpha_{\delta}}\right)\left(\eta\left(y, x_{\alpha_{\delta}}\right)\right) \rightarrow T(\bar{x}, \bar{z}, \bar{\lambda})(\eta(y, \bar{x})) .
$$

Thus,

$$
T\left(x_{\alpha_{\delta}}, z_{\alpha_{\delta}}, \lambda_{\alpha_{\delta}}\right)\left(\eta\left(y, x_{\alpha_{\delta}}\right)\right)+f\left(y, x_{\alpha_{\delta}}\right) \rightarrow T(\bar{x}, \bar{z}, \bar{\lambda})(\eta(y, \bar{x}))+f(y, \bar{x}) .
$$

Now, if

$$
T(\bar{x}, \bar{z}, \bar{\lambda})(\eta(y, \bar{x}))+f(y, \bar{x}) \in-\operatorname{int} C,
$$

then

$$
T\left(x_{\alpha_{\delta}}, z_{\alpha_{\delta}}, \lambda_{\alpha_{\delta}}\right)\left(\eta\left(y, x_{\alpha_{\delta}}\right)\right)+f\left(y, x_{\alpha_{\delta}}\right) \in-\operatorname{int} C
$$

eventually, which leads to a contradiction. Hence,

$$
T(\bar{x}, \bar{z}, \bar{\lambda})(\eta(y, \bar{x}))+f(y, \bar{x}) \notin-\operatorname{int} C,
$$

that is, $\bar{x} \in F(y)$.

Now, $F(y)$, being a closed subset of a compact set $K$, is compact. Therefore, by KKM-Theorem, $\bigcap_{y \in K} F(y) \neq \emptyset$. Hence, there exists $x_{0} \in F(y)$ for all $y \in$ $K$, that is, there exist $z_{0} \in K, \lambda_{0} \in[0,1]$, such that $T\left(x_{0}, z_{0}, \lambda_{0}\right)\left(\eta\left(y, x_{0}\right)\right)+$ $f\left(y, x_{0}\right) \notin-\operatorname{int} C$, for every $y \in K$.

In the next theorem, we provide the existence condition for the solution of $\eta$-GVVLIP (II).

Theorem 3.2. Let $\left(X, \tau_{1}\right)$ and $\left(Y, \tau_{2}\right)$ be two topological vector spaces and $\mathcal{C}(X, Y)$ be the space of all continuous linear mappings from $X$ to $Y$, equipped with an admissible topology. Let $K$ be a nonempty closed convex compact subset of $X$. Let $C: K \rightrightarrows Y$ be a set-valued map such that, for every $x \in K, C(x)$ is a closed convex pointed cone with $\operatorname{int} C(x) \neq \emptyset$. Suppose the set-valued map $W: K \rightrightarrows Y$ defined by $W(x)=Y \backslash(-\operatorname{int} C(x))$ has a closed graph $\mathcal{G}(W)$ in $X \times Y$. Let $T: K \times K \times[0,1] \rightarrow \mathcal{C}(X, Y)$ be a single-valued continuous mapping. Suppose the maps $\eta: K \times K \rightarrow X$ and $f: K \times K \rightarrow Y$ are affine mappings such that both are continuous in the second argument with $\eta(x, x)=0_{X}, f(x, x)=0_{Y}$, for all $x \in K$. Then, the $\eta$-GVVLIP (II) has a solution. That is, there exists $x_{0} \in K$ such that, for some $z_{0} \in K$ and for some $\lambda_{0} \in[0,1]$, the following holds

$$
T\left(x_{0}, z_{0}, \lambda_{0}\right)\left(\eta\left(y, x_{0}\right)\right)+f\left(y, x_{0}\right) \notin-\operatorname{int} C\left(x_{0}\right) \quad \forall y \in K .
$$


Proof. For each $y \in K$, we define a set-valued map $F: K \rightrightarrows K$ as $F(y)=\{x \in K: \exists z \in K, \exists \lambda \in[0,1]$ s.t. $T(x, z, \lambda)(\eta(y, x))+f(y, x) \notin-\operatorname{int} C(x)\}$. Clearly, for each $y \in K, F(y)$ is nonempty as at least $y \in K$. The proof of the theorem is divided into two steps:

(i) $F$ is a KKM-map on $K$;

(ii) $F(y)$ is closed for each $y \in K$.

We are avoiding the proof of step (i) as it is similar to that of Theorem 3.1.

Let $\left\{x_{\alpha}\right\}_{\alpha \in D}$ be a net in $F(y)$ converging to some $\bar{x} \in X$. As $K \subseteq X$ is closed, $\bar{x} \in K$. We have to show $\bar{x} \in F(y)$, that is, there exist some $\bar{z} \in K, \bar{\lambda} \in[0,1]$ such that

$$
T(\bar{x}, \bar{z}, \bar{\lambda})(\eta(y, \bar{x}))+f(y, \bar{x}) \notin-\operatorname{int} C(\bar{x}) .
$$

Since $x_{\alpha} \in F(y)$, therefore there exist nets $\left\{z_{\alpha}\right\}_{\alpha \in D}$ in $K$ and $\left\{\lambda_{\alpha}\right\}_{\alpha \in D}$ in $[0,1]$ such that $T\left(x_{\alpha}, z_{\alpha}, \lambda_{\alpha}\right)\left(\eta\left(y, x_{\alpha}\right)\right)+f\left(y, x_{\alpha}\right) \notin-\operatorname{int} C\left(x_{\alpha}\right)$, which implies

$$
T\left(x_{\alpha}, z_{\alpha}, \lambda_{\alpha}\right)\left(\eta\left(y, x_{\alpha}\right)\right)+f\left(y, x_{\alpha}\right) \in W\left(x_{\alpha}\right),
$$

which gives

$$
\left\{\left(x_{\alpha}, T\left(x_{\alpha}, z_{\alpha}, \lambda_{\alpha}\right)\left(\eta\left(y, x_{\alpha}\right)\right)+f\left(y, x_{\alpha}\right)\right)\right\} \in \mathcal{G}(W) .
$$

Now, following the lines of the proof of Theorem 3.1, we get subnets $\left\{x_{\alpha_{\delta}}\right\}_{\alpha_{\delta} \in D_{3}}$, $\left\{z_{\alpha_{\delta}}\right\}_{\alpha_{\delta} \in D_{3}}$ and $\left\{\lambda_{\alpha_{\delta}}\right\}_{\alpha_{\delta} \in D_{3}}$ of $\left\{x_{\alpha}\right\},\left\{z_{\alpha}\right\}$ and $\left\{\lambda_{\alpha}\right\}$, respectively, such that $\left\{x_{\alpha_{\delta}}\right\}$, $\left\{z_{\alpha_{\delta}}\right\},\left\{\lambda_{\alpha_{\delta}}\right\}$ converge to $\bar{x} \in K, \bar{z} \in K$ and $\bar{\lambda} \in[0,1]$, respectively.

As $T$ is continuous, $T\left(x_{\alpha_{\delta}}, z_{\alpha_{\delta}}, \lambda_{\alpha_{\delta}}\right) \rightarrow T(\bar{x}, \bar{z}, \bar{\lambda})$. Since the functions $\eta$ and $f$ are continuous in the second argument, therefore

$$
\eta\left(y, x_{\alpha_{\delta}}\right) \rightarrow \eta(y, \bar{x}), \quad f\left(y, x_{\alpha_{\delta}}\right) \rightarrow f(y, \bar{x}) .
$$

As the function space $C(X, Y)$ is admissible,

$$
T\left(x_{\alpha_{\delta}}, z_{\alpha_{\delta}}, \lambda_{\alpha_{\delta}}\right)\left(\eta\left(y, x_{\alpha_{\delta}}\right)\right) \rightarrow T(\bar{x}, \bar{z}, \bar{\lambda})(\eta(y, \bar{x})) .
$$

Thus,

$$
T\left(x_{\alpha_{\delta}}, z_{\alpha_{\delta}}, \lambda_{\alpha_{\delta}}\right)\left(\eta\left(y, x_{\alpha_{\delta}}\right)\right)+f\left(y, x_{\alpha_{\delta}}\right) \rightarrow T(\bar{x}, \bar{z}, \bar{\lambda})(\eta(y, \bar{x}))+f(y, \bar{x}) .
$$

The graph $\mathcal{G}(W)$ of $W$ is closed, therefore

$$
(\bar{x}, T(\bar{x}, \bar{z}, \bar{\lambda})(\eta(y, \bar{x}))+f(y, \bar{x})) \in \mathcal{G}(W),
$$

which implies

which leads to

$$
T(\bar{x}, \bar{z}, \bar{\lambda})(\eta(y, \bar{x}))+f(y, \bar{x}) \in W(\bar{x})
$$

Hence, $\bar{x} \in F(y)$.

Thus, for each $y \in K, F(y)$ is a closed subset of a compact set $K$, so $F(y)$ is compact. Now, by KKM-Theorem, we have $\bigcap_{y \in K} F(y) \neq \emptyset$. Hence, there exists $x_{0} \in K$ such that $x_{0} \in F(y)$, for all $y \in K$, that is, there exist $z_{0} \in K, \lambda_{0} \in[0,1]$ such that $T\left(x_{0}, z_{0}, \lambda_{0}\right)\left(\eta\left(y, x_{0}\right)\right)+f\left(y, x_{0}\right) \notin-\operatorname{int} C\left(x_{0}\right)$, for every $y \in K$.

Remark 3.3. The existence results for the solution of problems $\eta$-GVVLIP (III) and $\eta$-GVVLIP (IV) can be proved along similar lines to those of Theorem 3.1 and those of Theorem 3.2, respectively. 
Remark 3.4. If we take $\eta(x, y)=x-y, f(x, y)=0_{Y}$ for all $(x, y) \in K \times K, z=$ $0_{X}$ and $\lambda=0$, then the $\eta$-generalized vector variational-like inequality problems reduce to the vector variational inequality problems discussed in [11]. Also, we have

(i) Theorem 3.1 reduces to Theorem 3.1 of [11];

(ii) Theorem 3.2 reduces to Theorem 3.2 of [11].

Here, we provide an example to illustrate our results as well as to show that our results are independent of the result obtained by Li et al. [15].

Example 3.5. Consider $X=\mathbb{R}^{2}, Y=\mathbb{R}, K=[0,1] \times[0,1]$. Clearly, $K$ is closed convex and compact. Let $C: K \rightrightarrows Y$ be defined by $C(x)=\mathbb{R}^{+} \cup\{0\}$, for every $x \in K$. Then, $C(x)$ is a closed convex pointed cone with $\operatorname{int} C(x) \neq \emptyset$, and -int $C(x)=(-\infty, 0)$, for each $x \in K$. Let $\eta: K \times K \rightarrow X$ and $f: K \times K \rightarrow Y$ be defined by $\eta(y, x)=y-x$ and $f(y, x)=3(\|y\|-\|x\|)$, respectively. Further, let $T: K \times K \times[0,1] \rightarrow \mathcal{C}(X, Y)$ be defined by $T_{x}(u)=-\langle x, u\rangle$, where $x=\left(x_{1}, x_{2}\right)$ and $u=\left(u_{1}, u_{2}\right)$ are in $K$. That the induced topology of $\mathcal{C}(X, Y)$ is admissible can be verified by the fact that if $\left\{x_{n}\right\}$ converges to $x$ in $X$ and $\left\{h_{n}\right\}$ converges to $h$ in $\mathcal{C}(X, Y)$, then we have

$$
\begin{aligned}
\left\|h_{n}\left(x_{n}\right)-h(x)\right\| & =\left\|h_{n}\left(x_{n}\right)-h_{n}(x)+h_{n}(x)-h(x)\right\| \\
& \leq\left\|h_{n}\left(x_{n}\right)-h_{n}(x)\right\|+\left\|h_{n}(x)-h(x)\right\| \\
& \leq\left\|h_{n}\right\|\left\|x_{n}-x\right\|+\left\|h_{n}(x)-h(x)\right\| .
\end{aligned}
$$

Hence, $h_{n}\left(x_{n}\right) \rightarrow h(x)$.

We take $x_{0}=(0,0)$. Then, for any $y=\left(y_{1}, y_{2}\right), z=\left(z_{1}, z_{2}\right)$ in $K$, and $\lambda \in[0,1]$, we have

$$
\begin{aligned}
T_{\lambda x_{0}+(1-\lambda) z}\left(\eta\left(y, x_{0}\right)\right)+f\left(y, x_{0}\right) & =-\left\langle\lambda x_{0}+(1-\lambda) z, \eta\left(y, x_{0}\right)\right\rangle+f\left(y, x_{0}\right) \\
& =(\lambda-1)\left(z_{1} y_{1}+z_{2} y_{2}\right)+3\|y\| \geq 0
\end{aligned}
$$

for $z=\left(\frac{1}{2}, \frac{1}{2}\right), \lambda=\frac{1}{2}$ and for all $y \in K$. Therefore,

$$
T_{\lambda x_{0}+(1-\lambda) z}\left(\eta\left(y, x_{0}\right)\right)+f\left(y, x_{0}\right) \notin-\operatorname{int} C\left(x_{0}\right) .
$$

Hence, $x_{0}$ is a solution for the $\eta$-generalized vector variational-like inequality problem.

$T$ is not $\eta$-monotone in $C$ : Let $T: K \rightarrow \mathcal{C}(X, Y)$ and $\eta: K \times K \rightarrow K$ be two mappings and suppose $C=\bigcap_{x \in K} C(x) \neq \emptyset$. T is called $\eta$-monotone in $C([15])$ if and only if, for every pair $x, y \in K$, we have $\langle T(x)-T(y), \eta(x, y)\rangle \in C$.

Now,

$$
\langle T(x)-T(y), \eta(x, y)\rangle=T_{x-y}(\eta(x, y))=-\langle x-y, x-y\rangle=-\|x-y\|^{2}<0 .
$$

Thus, $T_{x-y}(\eta(x, y)) \notin C$. Hence, $T$ is not $\eta$-monotone in $C$.

In the following result, we discuss some topological properties of the solution sets obtained above.

Theorem 3.6. The solution set for the $\eta$-GVVLIP (I) (or $\eta$-GVVLIP (II)) obtained in Theorem 3.1 (or Theorem 3.2) is closed as well as compact. 
Proof. Let $F: K \rightrightarrows K$ be the set-valued map defined by

$$
F(u)=\{x \in K: \exists z \in K, \exists \lambda \in[0,1] \text { s.t. } T(x, z, \lambda)(\eta(u, x))+f(u, x) \notin-\operatorname{int} C\} .
$$

Then, by Theorem 3.1, the solution set $\mathcal{S}$ of the $\eta$-GVVLIP (I) is given by $\mathcal{S}=$ $\bigcap_{u \in K} F(u)$. As shown in Theorem 3.1 (or Theorem 3.2), $F(u)$ is closed for every $u \in K$. Therefore, $\bigcap_{u \in K} F(u)$ is closed, that is, $\mathcal{S}$ is closed. Also, $\mathcal{S}$, being a closed subset of a compact set $K$, is compact.

\section{CONCLUSION}

In this study, we have provided existence theorems for the solution of two variants of the $\eta$-generalized vector variational-like inequality problem by adopting a topological approach, a significantly different one from those in the existing literature so far. The admissibility of function space topology and net theory are the major tools of achieving the main results. These tools have not been extensively used earlier in literature to obtain such results. It would be interesting to see whether this approach may be used for other variants of variational inequality problems.

Acknowledgement. The authors sincerely thank the referee for his/her valuable suggestions, which helped improve the quality of the paper.

\section{REFERENCES}

[1] R. Arens and J. Dugundji, Topologies for function spaces, Pac. J. Math. 1 (1951), 5-31.

[2] K. Fan, Some properties of convex sets related to fixed point theorem, Math. Ann. 266 (1984), 519-537.

[3] A. P. Farajzadeh and B.S. Lee, Vector variational-like inequality problem and vector optimization problem, Appl. Math. Lett. 23 (2010), 48-52.

[4] F. Giannessi, Theorems of alternative, quadratic programs and complementarity problems, in: R. W. Cottle, F. Giannessi and J. L. Lions (eds.), Variational Inequalities and Complimentary Problems, John Wiley and Sons, Chichester, U.K., 1980, pp. 151-186.

[5] A. Gupta, S. Kumar, R. D. Sarma, P. K. Garg and R. George, A note on the generalized non-linear vector variational-like inequality problem, J. Func. Spaces, 2021 (2021), Article ID 4488217,7 pp.

[6] A. Gupta and R. D. Sarma, A study of function space topologies for multifunctions, App. Gen. Topol. 18 (2017), 331-344.

[7] M. A. Hanson, On sufficiency of the Kuhn-Tucker conditions, J. Math. Anal. Appl. 80 (1981), 545-550.

[8] S. A. Khan, On set-valued mixed vector variational-like inequalities in Banach spaces, J. Nonlinear Anal. Optim. 3 (2012), 255-267.

[9] S.A. Khan, Vector variational-like inequalities with generalized semimonotone mappings, Int. J. Anal. 2013 (2013), Article ID 762380, 7 pp.

[10] S. A. Khan, Q. H. Khan and F. Suhel, Generalized vector mixed variational-like inequality problem without monotonicity, Thai J. Math. 10 (2012), 245-258.

[11] S. Kumar, A. Gupta and P. K. Garg, Topological variants of vector variational inequality problem, presented in International Conference on Sustainable development: Modelling and Optimization (ICSDMO), Feb 16-17, 2021.

[12] G. M. Lee, On relations between vector variational inequality and vector optimization problem, in: X. Yang et al. (eds.), Progress in Optimization, Contributions from Australasia, Applied Optimization 39, Kluwer, 2000, pp. 167-179.

[13] B.-S. Lee, M. Firdosh Khan and Salahuddin, Generalized vector variational-type inequalities, Comput. Math. Appl. 55 (2008), 1164-1169. 
[14] G. M. Lee, D. S. Kim, B. S. Lee and N.D. Yen, Vector variational inequality as a tool for studying vector optimization problems, Nonlinear Anal. 34 (1998), 745-765.

[15] X. Li, J. K. Kim and N.-J. Huang, Existence of solutions for $\eta$-generalized vector variationallike inequalities, J. Inequal. Appl. 2010 (2010), Article ID 968271, 13 pp.

[16] R. Li and G. Yu, A class of generalized invex functions and vector variational-like inequalities, J. Inequal. Appl. 2017 (2017), Paper No. 70, 14 pp.

[17] H. Q. Ma, N. J. Huang, M. Wu and D. O'Regan, A new gap function for vector variational inequalities with an application, J. Appl. Math. 2013 (2013), Article ID 423040, 8 pp.

[18] S. K. Mishra and M. A. Noor, On vector variational-like inequality problems, J. Math. Anal. Appl. 311 (2005), 69-75.

[19] M. A. Noor On generalized preinvex functions and monotonicities, J. Inequal. Pure Appl. Math. 5 (2004), Paper No. 110, 9 pp.

[20] G. Ruiz-Garzón, R. Osuna-Gómez and A. Rufián-Lizan, Relationships between vector variational-like inequality and optimization problems, Eur. J. Oper. Res. 157 (2004), 113119.

[21] Salahuddin, General set-valued vector variational inequality problems, Commun. Optim. Theory 2017 (2017), Article ID 13, 16 pp.

[22] Salahuddin, Solutions of vector variational inequality problems, Korean J. Math. 26 (2018), 299-306.

[23] G. Stampacchia, Formes bilinéaires coercitives sur les ensembles convexes, C. R. Acad. Sci., Paris 258 (1964), 4413-4416.

[24] D. E. Ward and G. M. Lee, On relations between vector optimization problems and vector variational inequalities, J. Optim. Theory Appl. 113 (2002), 583-596.

[25] T. Weir and B. Mond, Pre-invex functions in multiple objective optimization, J. Math. Anal. Appl. 136 (1988), 29-38.

[26] T. Xie and Z. Gong, Variational-like inequalities for $n$-dimensional fuzzy-vector-valued functions and fuzzy optimization, Open Math. 17 (2019), 627-645.

[27] L. Xie, J. Li and W. Yang, Vector and ordered variational inequalities and applications to order-optimization problems on Banach lattices, J. Appl. Math. 2013 (2013), Article ID 439394, 7 pp.

[28] X. Q. Yang, Vector variational inequality and vector pseudolinear optimization, J. Optim. Theory Appl. 95 (1997), 729-734.

[29] X. Q. Yang and C. J. Goh, On vector variational inequalities: Application to vector equilibria, J. Optim. Theory Appl. 95 (1997), 431-443.

[30] J. C. Yao, Applications of variational inequalities to nonlinear analysis, Appl. Math. Lett. 4 (1991), 89-92.

Satish Kumar, Department of Mathematics, University of Delhi, Delhi 110007, India e-mail: satishataria47@gmail.com

Ankit Gupta, Department of Mathematics, Bharati College, University of Delhi, Delhi 110058, India

e-mail: ankitsince1988@yahoo.co.in

Pankaj Kumar Garg, Department of Mathematics, Rajdhani College, University of Delhi, Delhi 110015, India

e-mail: gargpk08@gmail.com

Ratna Dev Sarma, Department of Mathematics, Rajdhani College, University of Delhi, Delhi 110015, India

e-mail: ratna_sarma@yahoo.com 
